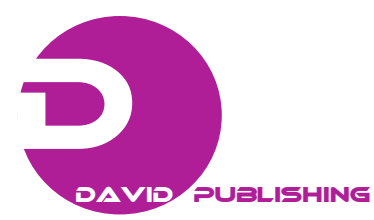

\title{
Rapid Detection of Salmonella in Foods Using PCR-Based Detection Kit
}

Teresita J. Ramirez ${ }^{*}$, Ma. Teresa M. Perez ${ }^{1}$, Susan A. Sedano ${ }^{1}$, Arsenia B. Sapin ${ }^{1}$, Rachel R. Elano ${ }^{2}$, Lydia S. Manguiat $^{3}$, Mylele L. Bool ${ }^{4}$, Cyril M. Madrid ${ }^{5}$, Elizabeth C. Conde ${ }^{6}$ and Ruby J. Apilado ${ }^{7}$

1. National Institute of Molecular Biology and Biotechnology (BIOTECH), University of the Philippines Los Baños (UPLB), College, Laguna

2. Food Development Center, FTI Complex, Taguig, Metro Manila

3. Regional Standard and Training Laboratory, DOST Region IV, Los Baños, Laguna

4. Philippine National Collection of Microorganisms, UP Los Baños, College, Laguna

5. Progressive Laboratories, 149 Dangay St., Project 7, Quezon City

6. Lipa Quality Control Center, Lipa City, Batangas

7. Food and Nutrition Research Institute, DOST, Taguig City

*Corresponding author's e-mail: tessjram@yahoo.com

\begin{abstract}
The current study validates a rapid PCR-based detection method and developed at BIOTECH-UPLB in comparison with the traditional AOAC/BAM culture method involving enrichment step and plating in selective media. The kit consisted of reaction tubes containing selected primer set for Salmonella. The protocol includes two-step enrichment period, DNA extraction and amplification of DNA in a thermal cycler followed by gel electrophoresis procedure. Validation of the detection kit was done on both naturally-contaminated processed and artificially-spiked food samples with the assistance of some government and private institutions. Results from 185 non-spiked samples categorized into 14 groups revealed 100\% agreement in all the sample categories except with flours, gums and gelatin (90\%). This gives specificity value of 1.00 in all food groups except with flours, gums and gelatin (0.86). Of the 459 samples analyzed, perfect agreement was obtained in both high and low inoculum levels in the following food groups/categories: coconut and processed coconut products, dairy products, flours, gums and gelatin and spices. Significant difference $\left(x^{2}>3.84\right)$ was observed only for the following food groups/categories with low inoculum: bakery products; egg and egg products; and for both high and low inoculum levels for processed fish and sea foods. The kit has sensitivity of $10^{4}$ cells per mL. Results obtained from the nine collaborating laboratories with 314 total samples analyzed showed agreement values ranging from $81.25 \%$ to $100 \%$, with no significant difference between the two methods employed. The kit can be recommended for food products found in this study to be compatible with Salmonella DAS ${ }^{\mathrm{TM}}$ kit and result can be obtained within 26-28 h. The PCR-based kit produced definite results without any confirmatory tests and is relatively easy to use over the culture method. The shorter analysis time makes the kit ideal for use in food industry.
\end{abstract}

Key words: Salmonella, PCR-based kit, DNA-based kit. 